\title{
Die Veränderungen des kolloid=osmotischen Drucks und der Eiweissfraktion des Blutserums bei akuter Kohlenoxydvergiftung.
}

(Studien über den kolloid-osmotischen Druck des Blutes im normalen und pathologischen Zustand. XXII.)

\author{
Von
}

Hiroshi Miura.

(三浦弘)

(Aus der Medizinischen Klinik von Prof. Dr. T. Kato, Tohoku Reichsuniversität zu Sendai.)

Über die Veränderungen des Stickstoffstoffwechsels bei der akuten Kohlenoxyd vergiftung liegen meines Wissens nur einige Angaben vor. Fränkel, ${ }^{17}$ Araki, ${ }^{2)}$ Panton u. Eason ${ }^{3)}$ u. a. haben im Tierversuch bei betreffender Vergiftung eine Zunahme der N-Ausscheidung konstatiert, Münzen u. Palma haben auf eine enorm gesteigerte Ausscheidung der Harnsäure hingewiesen. Wolf u. Österbe $\mathrm{rg}^{5)}$ berichteten darüber, dass sich eine Zunahme der Kreatinausscheidung findet, die aber nicht konstant auftritt. Büttner ${ }^{6}$ hat experimentell nachgewiesen, dass bei der Leuchtgasvergiftung der Rest- $\mathrm{N}$ des Blutes eine Verminderung erfährt. Über die Veränderungen des Bluteiweisses, welche bei der CO-Vergiftung auftreten, sind Untersuchungen bisher so gut wie nicht veröffentlicht worden.

Nun wollen wir einen Rückblick auf das Wesen der akuten COVergiftung werfen. Es stehen derzeitig darüber zwei Theorien gegenüber. Die eine Theorie erblickt das Wesen der Vergiftung in direkten oder indirekten Einflüssen der allgemeinen Anoxämie, welche durch die Abnahme des Hämoglobins-weil bekanntlich das Koh-

\footnotetext{
1) Fränkel, Virchow's Arch., 1876, 67, 273.

2) Araki,Hop pe-Seyler's Ztschr., 1894, 19, 422.

3) Panton u. Eas on, Journ. Physiol, 1900-1901, 26, 166.

4) Münzen u. Pa Ima, Ztschr. f. Heilk., 1894, 15, 189.

5) Wolf u. Oesterberg, Biochem. Ztschr., 1909, 16, 476.

6) Büıtner, Ztschr. exp. Med., 1933, 88, 363.
} 
lenoxyd gegenüber dem Sauerstoff eine weitaus stärkere Affinität zum Hämoglobin, dem die Aufgabe als $\mathrm{O}_{2}$-Transporter zufällt, besitzt und deshalb das Hämoglobin begierig an sich reisst,- - ausgelöst wird. Diese Theorie ist erstmalig von $\operatorname{Araki}^{7^{7}}$ und $\mathrm{Haldan \textrm {e } ^ { \mathrm { g } ) }}$ vertreten und in der Folge durch Untersuchungen von $\mathrm{Haggard},{ }^{9)}$ Boer u. Carrol, ${ }^{10)}$ Chevallier u. Desoilles ${ }^{11)}$ u. a. bestätigt worden. Campbel1 ${ }^{12)}$ hat den Beweis geliefert, dass zwischen den Sektionsbefunden des von der chronischen CO-Vergiftung betroffenen Tieres und des in sauerstoffarmer pneumatischer Kammer gehaltenen Tieres keine Differenz besteht. Altschul ${ }^{13)}$ hat die Veränderungen im Zentralnervensystem bei Erstickung und akuter CO-Vergiftung gegeneinander verglichen und fand, dass die. Veränderungen sich miteinander äusserst ähnlich verhalten. Andere Schule will als das Wesen der CO-Vergiftung zur Anoxämie noch die spezifische toxische Wirkung von CO hinzugereicht wissen. Als Urbeber dieser Ansicht ist Geppert $t^{14)}$ zu nennen; er nimmt die toxische Wirkung des Kohlenoxyds auf das Atemzentrum an. Wolf $u$. Österberg ${ }^{5)}$ vermuten das Vorkommen irgendeines giftigen Einflusses im Eiweissstoffwechsel. Barkan ${ }^{15}$, hebt hervor, dass die CO-Vergiftung: schlechthin nicht als die einfache Anoxämie anzusehen sei. Aus den Sektionsbefunden bei Menschen und im Tierversuch wollen Hiller, ${ }^{16)}$ Weimann, ${ }^{17}$ Meyer ${ }^{18)}$ u.a. die toxische Wirkung des CO auf den gesamten Kreislauf an nehmen. Wie die Dinge hierbei auch liegen mögen, ist bei der CO-Vergiftung allerdings dem $\mathrm{O}_{2}$-Mangel eine äusserst wichtige Bedeutung beizumessen.

Es erhebt sich dann die Frage, welche Veränderungen denn das Bluteiweiss, wenn der Organismus in den Zustand des $\mathrm{O}_{2}$-Mangels verfällt, erleidet. Es liegen diesbetreffend folgende Angaben vor.

Frenkel-Tissot ${ }^{19)}$ gibt an, dass in einer $1700-2000 \mathrm{~m}$ Höhe das Serum-

7) Araki, Hoppe.Seyler's Ztsehr., 1891, 15, 335.

8) Hald a ne, Journ. Physiol., 1895, 18, 201.

9) Haggard, Amer. Journ. Physiol., 1921, 56, 390 u. 1922, 60, 244.

10) Boer u. Carrol, Journ. Physiol., 1924-25, 59, 312.

11) Chevallier u. Desoilles, Ann. d. Méd., 1930, 28, 56.

12) Ca m p bell, Brit. Journ. Exp. Path., 1929, 10, 304.

13) Altschul. Mschr. f. Psychiat. u. Neurol., 1931, 80, 343.

14) Geppert, Dtsch. med. Wschr., 1892, 418.

15) Barkan, Bethe's Handbuch der normalen u. pathologischen Physiologie, Bd. 6, 1. Hälfte, Berlin 1928, 138.

16) Hille r, Ztschr. f. ges. Neurol. a. Psychiat., 1924, 93, 694.

17) We i m a n, Ibid., 1926, 105, 213.

18) Me yer, Ibid., 1926, 100, 201.

19) Frenkel-Tis sot, Münch. med. Wschr., 1921, 1616. 
eiweiss, speziell Globulin abnimmt. Toth $\mathrm{h}^{20)}$ sab bei Einatmung von verdünter Luft bald eine Zunahme, bald aber auch eine Abnahme des Globulins. Schmensky ${ }^{21}$ beobachtete beim Aufenthalt im Hochgebirge die Globulinabnahme und die Erniedrigung der Blutviskosität. Elias u. Taubenhaus ${ }^{22}$ haben experimentell nachgewiesen, dass beim Kaninchen, welches unter Unterdruck einige Tage gehalten worden ist, das Globulin entweder gleichbleibend oder herabgesetzt ist, während das Albumin zunimmt. Loew y ${ }^{23)}$ beschreibt in seinem Monograph, dass das Bluteiweiss im Höhenklima mancherlei Veränderungen aufweist. Kasuga ${ }^{24)}$ an hiesiger Klinik hat an Hunden, welche die $\mathrm{O}_{2}$-arme Luft eingeatmet haben, eine Zunahme des Gesamt-N sowie des Serumeiweisses, besonders Globulins und eine Abnahme des kolloid-osmotischen Drucks pro $1 \%$ Eiweiss nachgewiesen.

Nach obigen literarischen Angaben lässt es sich ohne weiteres vermuten, dass bei der akuten CO-Vergiftung das Bluteiweiss qualitativ und quantitativ bestimmte Veränderungen erfährt. Um dies experimentell zu beweisen, habe ich an Tieren die Konzentration und Fraktionen des Bluteiweisses, den Rest-N sowie den kolloid-osmotischen Druck des Blutes vor und nach Einatmung von $\mathrm{CO}$ bestimmt.

Versuchsmethodik. Als Versuchstiere wurden ausgewachene, gesunde Kaninchen benutzt. Dabei liess ich Versuchstiere die CO-haltige Luft nach gleicher Methode, wie sie in früher Mittei-

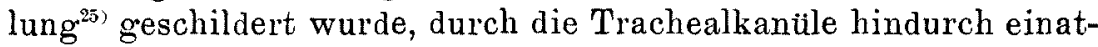
men; die Blutproben wurden in allen Fällen aus der in die A. femoralis eingeführten Kanüle entnommen. Der kolloid-osmotischer Druck des Serums (k.o.D.) wird nach der Methode von Krogh u. $\mathrm{Nakazaw} \mathrm{a}^{26}$. bestimmt und mit $\mathrm{mm} \mathrm{H}_{2} \mathrm{O}$ ausgedrückt, und wenn man hier erhaltene $\mathrm{Zahlen}$ von $\mathrm{mm}_{2} \mathrm{O}$ durch den mittels des Refraktometers $\mathrm{Pulf}$ ric h s bestimmten Eiweissgehalt dividiert, so ergibt sich k. o. D. pro 1\% Eiweiss (Dr. pro \%). Die Eiweissfraktionen wurden mit dem Parnas-Wagner'schen ${ }^{27)}$ Apparat nach der Methode von $\mathrm{H}$ ow $\mathrm{e}^{28}$ bestimmt. Im übrigen wurde der NaCl-Gehalt des Serums nach der Ruszny ak'schen $\left.{ }^{29}\right)$ Mikromethode, der Erythrozytendurch-

20) Toth, Biochem. Ztschr., 1928, 201, 412.

21) Schmensky, Ztschr. f. kl. Med., 1929, 111, 205.

22) Elias u. Taubenhaus, Ztschr. f. exp. Med., 1930, 74, 69.

23) Loewy, Physiologie des Höhenklimas, Berlin 1932, 97.

24) Kasugai, Tohoku Journ. Exp. Med., 1935, 27, 487 u, 505.

25) Miura, Tohoku Journ. Exp. Med., 1936, 29, 686.

26) Krogh u. Na k a a wa, Biochem. Ztschr., 1927, 188, 241.

27) Parnas-Wagner, Ibid,, 1921, 125, 258.

28) Howe, Journ. Biol. Chem., 1921, 49, 93 n. 109.

29) Rusznyak, Biochem. Ztschr., 1921, 114, 23. 
messer mit dem Bockschen Erythrozytometer, ${ }^{30}$ ) der Hämoglobingehalt mit dem Fle is chl-Mie s ch e r'schen Hämometer bestimmt und die Erythrozytenzahl mittels der Thoma-Zeiss'schen Zählkammer gezählt.

\section{Die Veränderungen des k.o.D. des Blutserums bei Einatmung von kohlenoxydhaltiger Luft.}

Kontrollversuch.

Als Kontrollversuch zur Untersuchung über den Einfluss des CO auf den k. o. D. des Blutes habe ich gesunde Kaninchen die atmosphärische Luft aus dem Spirometer 60-80 Minuten lang einatmen lassen und ihnen zu 3-4 Malen je ca. 1,7 ccm Blut entnommen. An einzelnen Blutportionen wurde der Eiweissgehalt des Blutes und der k. o.D. des Serums bestimmt, um darüber zu untersuchen, ob und inwieweit die Atmung aus dem Spirometer und mehrmalige Blutentnahmen das Serumeiweiss bei gesunden Kaninchen beeinflussen könnten.

Diesbeziigliche Data sind in Tab. 1 wiedergegeben.

Serumeiweiss, k. o.D. sowie Dr. pro \% erfuhren entsprechend der Häufigkeit der Blutentnahme gemeinschaftlich immer grössere Abnahmen, welche jedoch nicht so erheblich waren, es sei denn, dass die Abnahme des k.o.D. mehr oder weniger stärker war. Nach experimentellen Untersuchungen von $\mathbf{F}$ is ch be r $\mathrm{g}^{312}$ und $\mathrm{S}$ a i t $\mathrm{o}^{32}$ dürften derartige Abnahmen von der geringfügigen Anämie durch die Blutentnahme und der unphysiologischen Atmung aus dem Spirometer herrühren.

\section{CO-Versuch.}

$\mathrm{Da}$ ich anfangs durch das Einatmenlassen von 0,2\% CO-haltiger Luft keine merkliche Veränderung wahrnehmen konnte, habe ich den CO-Gehalt der Einatmungsluft auf 0,5-1,0\% erhöht und dieses Gasgemisch Kaninchen 30-90 Minuten lang einatmen lassen.

Die Ergebnisse sind in Tab. 2 zusammengestellt.

Nach den Werten, welche nach 60-80 Minuten lang dauernder Einatmung von oben erwähnter abnormer Luft ermittelt wurden,

30) Bock, Kl. Wschr., 1933, 1141.

31) Fisch berg, Journ. Biol. Chem., 1929, 81, 205.

32) Saito, Tohoku Journ. Exp. Med., 1932, 19, 445 u. 462. 


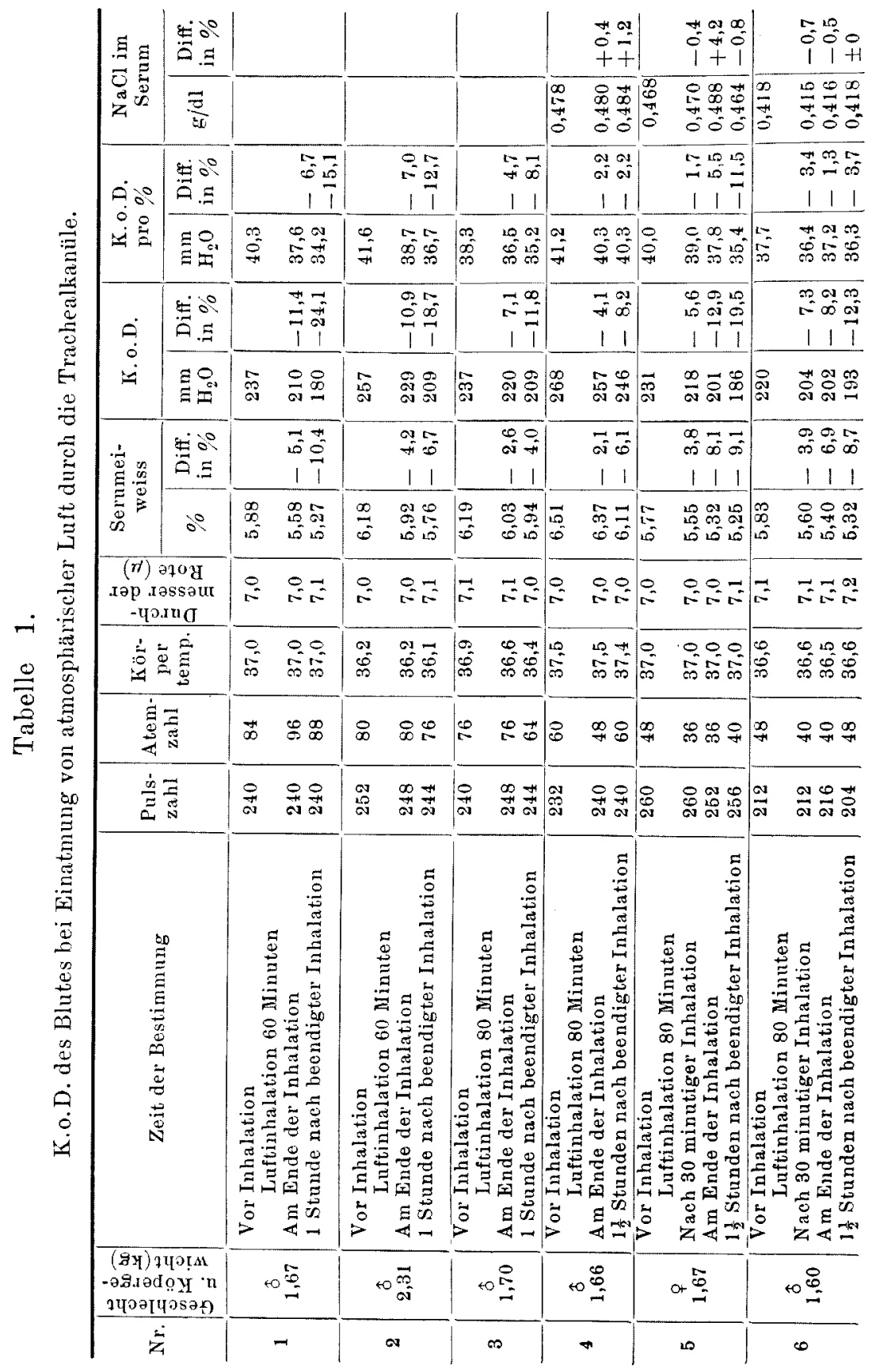




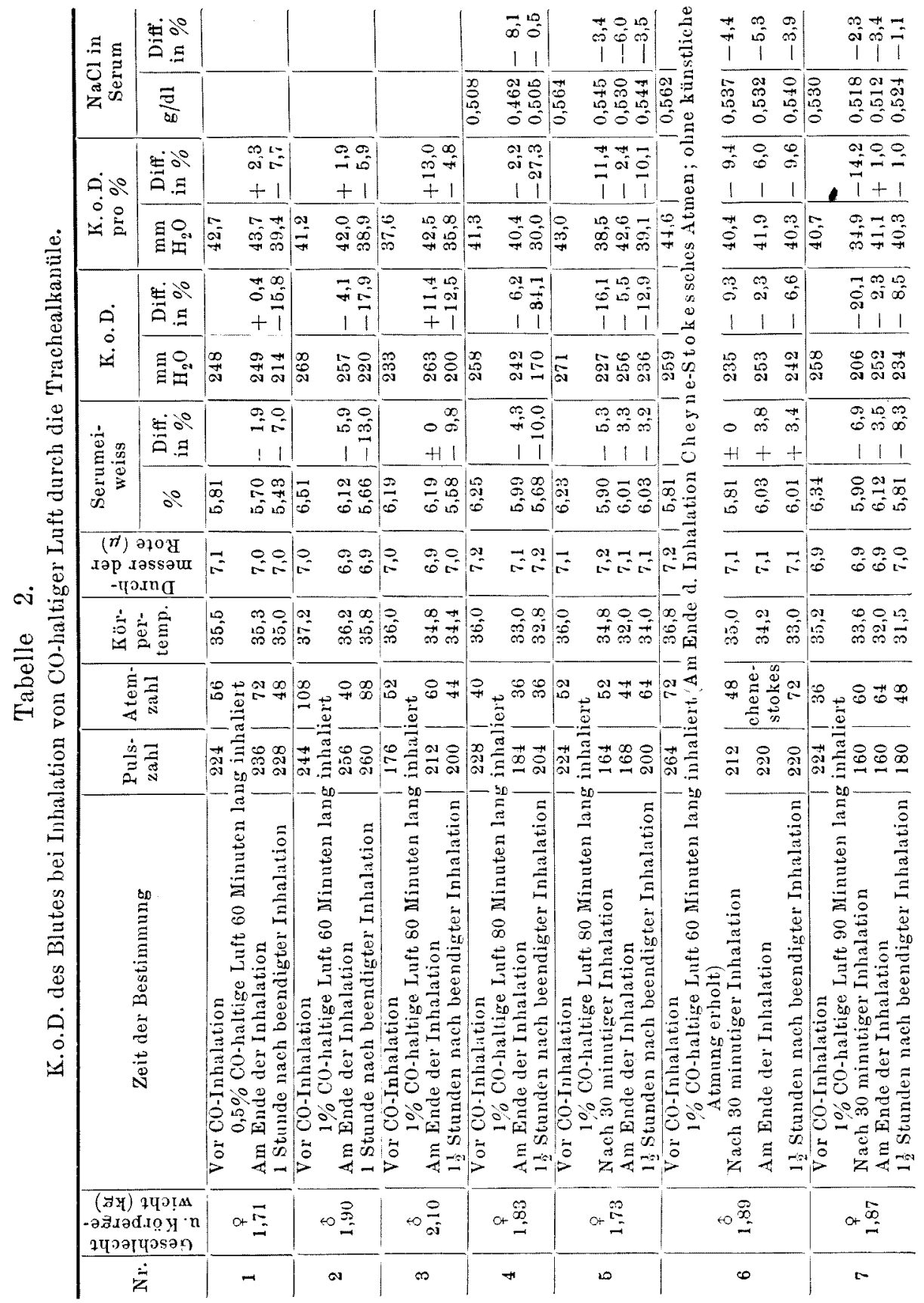


beurteilt, zeigte das Serumeiweiss (Versuch 1-4) eine geringe Abnahme, welche von derselben im Kontrollversuch nicht so sehr abwich. Zu gleicher Zeit zeigte k.o.D. teils eine geringe Erniedrigung, teils umgekehrt eine Erhöhung. Derartiges unter sich divergierende Verhalten des k. o. D. unterscheidet sich deutlich von demselben im Kontrollversuch, wo k.o.D. gleichmässig absank. Der Dr. pro \% nahm in 3 Fällen zu, in einem einzigen Fall nur in geringem Masse $a b$; diese Abnahme war jedoch nicht so stark wie im Kontrollversuch.

Greifen wir auf die Veränderungen (in Versuch 5-7), welche mitten unter fortlaufender Einatmung auftraten, zurück. 30 Minuten nach Beginn der Einatmung von 1\% CO-haltiger Luft erfuhren Serumeiweiss, k.o.D. und Dr. pro \% erhebliche Abnahmen, welche stärker waren als die an den zu gleicher Zeit entnommenen Kontrollblutproben ermittelten Werte; besonders ausgeprägt war die Abnahme bei k. o. D. sowie Dr. pro \%. Am Ende der Inhalation (6080 Minuten) zeigten absolute Werte der beiden im Vergleich zu den bei halbstündiger Inhalation ermittelten Werten deutliche Zunahme. Diese Zunahme war besonders augenfällig beim Kaninchen (Versuch 6), das gegen das $\mathrm{CO}$ anscheinend stärkere Empfindlichkeitslage besass, und bei welchem deswegen schon 60 Minuten nach Beginn der Einatmung von 1\% CO-haltiger Luft das Cheyne-Stokessche Atemphänomen beobachtet wurde, indem das Serumeiweiss noch höhere Werte als vor der Inhalation aufwies und selbst mitten unter fortlaufender Einatmung nie abnahm. Derartiges Verhalten des Serumeiweisses dürfte so gedeutet werden, dass das Serumeiweiss schon 30 Minuten vor der erfolgten Bestimmung einmal abgenommen hatte, und gerade zu einer Zeit, wo es wieder zuzunehmen anfing, bestimmt wurde. Auch in Versuch 7 wurde Dr. pro \% am Ende der Inhalation mehr vermehrt gefunden als vor der Inhalation.

Überblickt man über einzelne, 60-90 Minuten nach Abschluss der. Inhalation ermittelte Werte in jedem Fall, so ersieht man, dass Serumeiweiss, k.o.D. sowie Dr. pro \%, welche alle im vorgeschrittenen Stadium der Vergiftung die Tendenz zur Zunahme aufgewiesen hatten, nach Abschluss der Inhalation wieder rasch herabgesetzt waren und, ausgenommen den durch Vergiftung moribund gewordenen Versuch 6 , schliesslich im ganzen genommen, annähernd gleiche Werte, wie die an den Blutproben aus solchem Kaninchen, welches die CO-haltige Luft nicht eingeatmet hat, erhaltenen Werte, zeigten. 
Oben erwähnte Ergebnisse können dahin zusammengefasst werden: Serumeiweiss, k.o.D. und Dr. pro \% erfahren im Anfangsstadium der CO-Vergiftung einmal erhebliche Abnahmen und schlagen mit fortschreitender Vergiftung ins Gegenteil, also in die $\mathrm{Zu}$ nahme um.

Der zu gleicher Zeit bestimmte Erythrozytendurchmesser zeigte die Veränderung nicht in dem Masse, als sie mit dem Bock'schen Apparat gemessen werden könnte; auch der $\mathrm{NaCl}$-Gehalt des Blutserums nähm mehr oder weniger $a b$, jedoch in unerheblichem Masse. Kubo u. Mitsui ${ }^{33)}$ und Sasabu ${ }^{34)}$ konnten bei langsam eintretender Asphyxie eine Abnahme des Serum-NaCl-Gehaltes nachweisen.

\section{Die Veränderungen der Eiweissfraktionen des Blutserums bei Einatmung von kohlenoxydhaltiger Luft.}

Kontrollversuch.

5 Kaninchen hat man die blosse atmosphärische Luft, welche kein CO enthielt, aus dem Spirometer 60-80 Minuten lang einatmen lassen und vor Beginn und am Ende der Inhalation sowie 60-80 Minuten nach Abschluss derselben, im ganzen also dreimal je ca. $3 \mathrm{ccm}$ arterielles Blut aus A. femoralis entnommen und an diesen Blutportionen die Fraktionen des Serumeiweisses bestimmt, um so erhaltene Werte als Kontrolle für den nachträglich ausgeführten CO-Versuch zu brauchen.

Die hierbei erhobenen Data sind in Tab. 3 zusammengestellt.

Hämoglobin, Erythrozytenzahl und Gesamt-N nahmen mit der Häufigkeit der Blutentnahme fast parallel immer stärker ab; allein der Rest-N nahm bald zu, bald ab, weswegen er keine regelrechte Veränderung erkennen liess. Der Albumin-N (Alb.-N) verminderte sich in erheblichem Masse, doch graduell. Globulin-N (Glob.-N) vermehrte sich, wenn es auch darunter einige Ausnahmefälle gab, im wesentlichen dem zunehmenden Quantum des entnommenen Blutes entsprechend. Das Albumin-Globulinmischverhältnis, also der Eiweissquotient (Eiw.-Quot.) nahm mithin in umgekehrtem Verhältnis zu entnommenen Blutmengen ab.

$\mathrm{Kasugai^{24 }}$ ) hat die Beobachtung gemacht, dass bei Hunden eben angeführte Grössen durch fortgesetzte Blutentnahme allein unverändert bleiben;

33) Kubo u. Mitsui, Japan Medical World, 1928, 8, 259.

34) S a s a u, Hokkaido Igaku Zasshi, 1929, 7, 1689. 


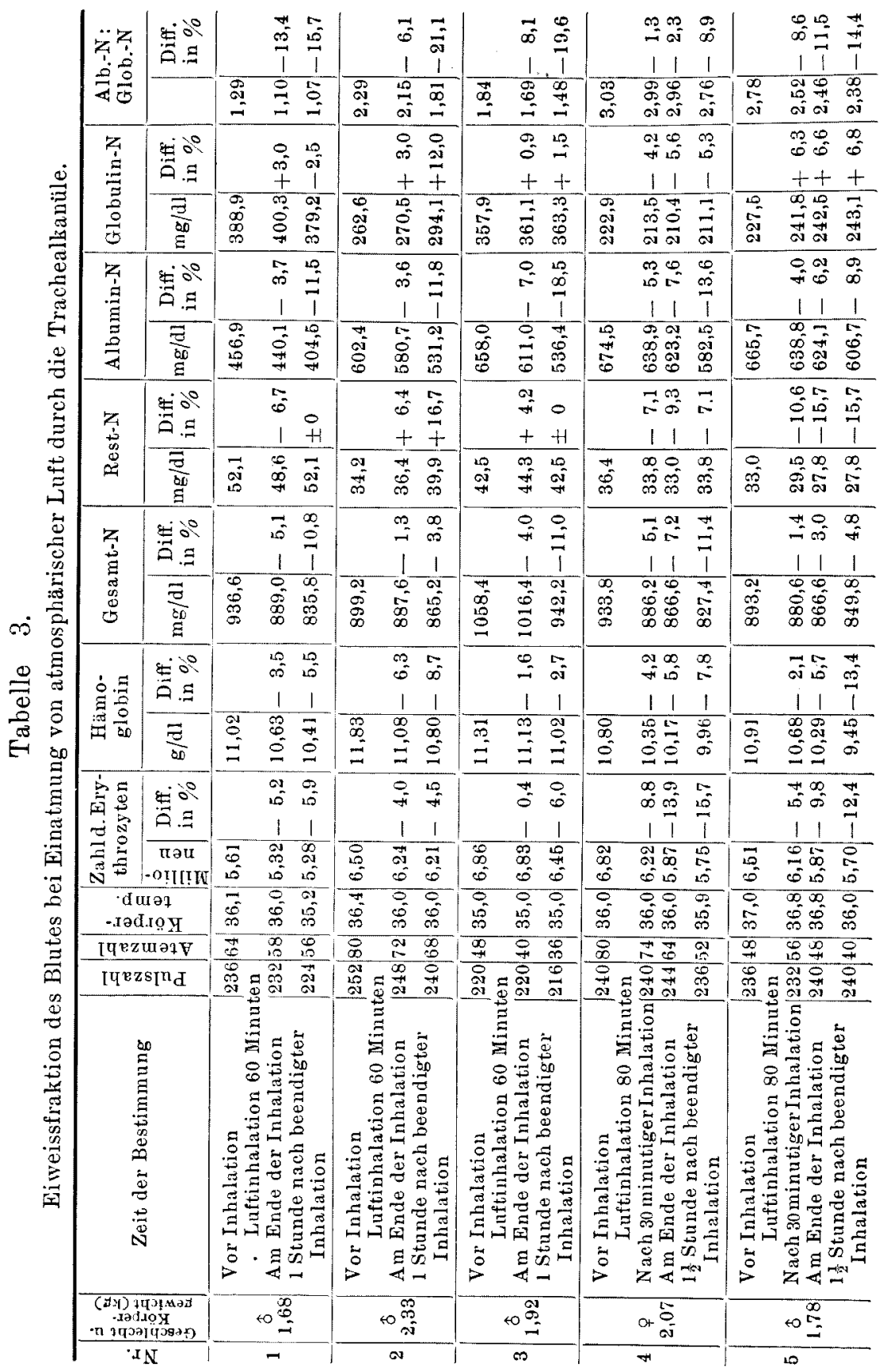


aber es ist leicht anzunehmen, dass bei Kaninchen gleiche Blutentnahme wegen des bescheidenen Körpergewichtes auf den Eiw.-Quot. stärkere Einflüsse ausubt. It $o^{35}$ hat experimentell nachgewiesen, dass bei Kaninchen die häufige Blutentnahme auch in jeweils kleinen Mengen, zu allmählichen Abnahmen des Hämoglobins, Serumeiweisses und des Gesamt-N führt. Angesichts der Versuchsergebnisse von Bodansky $y^{36}$ u. a., Hashimoto u. Goto ${ }^{3 i}$ und Fishberg, ${ }^{38}$ denen zufolge bei experimenteller Anämie die Abnahme des Gesamt$\mathrm{N}$, vor allem aber die Verminderung des Alb.- $\mathrm{N}$ im Verhälnis zum Glob.- $\mathrm{N}$ in erheblichem Masse erfolgt, dürfte die ron mir ermittelte Veränderung der Eiweissfraktion im direkten Zusammenhang mit Blutentnahmen stehen.

\section{CO-Versuch.}

Da durch Einatmung von 0,5\% CO-haltiger Luft keine nennenswerte Veränderung konstatiert werden konnte, habe ich in den meisten Fällen die 1\% CO-haltige Luft einatmen lassen.

Die Ergebnisse der Versuche über die Einatmung von CO-haltiger Luft, wo die Blutentnahme und sonstige Verfahren unter ganz gleichen Bedingungen wie in eben besprochenem Kontrollversuche vorgenommen wurden, sind in Tab. 4 wiedergegeben.

$\mathrm{Hb}$-Gehalt, Erythrozytenzahl und Gesamt-N usw. erfuhren mit zunehmendem Quantum des entnommenen Blutes allmähliche $\mathrm{Ab}$ nahmen. In Versuch 7 wurden die Werte, die am Versuchsende (90 Minuten) ermittelt wurden, mehr vermehrt gefunden als die Werte, die 30 Minuten nach Versuchs beginn bestimmt wurden.

Die Tatsache, dass bei protrahierter CO-Wirkung die Erythrozytenzahl und der $\mathrm{Hb}$-Gehalt zunehmen, scheint durch Untersuchungen von $\mathrm{Nasmith}$. Graham, ${ }^{39)} \mathrm{Nishimura},{ }^{40)}$ Horiki, ${ }^{41)}$ Tameno, ${ }^{42)}$ Litzner ${ }^{43)}$ u.a. endgültig dargetan zu sein; dagegen erhob Ishikawa ${ }^{44}$ Einwände, dass derartige Veränderung kein charakteristisches Phänomen für die CO-Vergiftung sei. Sasada u. Kita$\mathrm{mu} 1^{45}$ haben darauf hingewiesen, dass durch die einmalige $\mathrm{CO}$ -

35) It o, Tohoku Journ. Exp. Med, 1929, 14, 198. 463.

36) Bodansky, Morse, Kiech u. Bramkam p, Journ. Biol. Chem., 1927, 74,

37) Hashimoto u. Goto, Nippon Naikagakkai Zasshi, 1929, 17, 250.

38) Fishberg, Biochem. Ztschr., 1928, 195, 20.

39) Nasinith u. Graham, Journ. Physiol, 1906-07, 35, 32.

40) Nishim ura, Kokumin Eisei, 1926-27, 4, 193.

41) Horiki, Ibid., 1928, 5, 517.

42) Tameno, Ibid., 1929, 6, 1194.

43) Litzner, Arch. f. Gewerbepath, u. Gewerbehyg., 1930, 1, 749.

44) Ishikawa, Kokka Igakkai Zasshi, $1912,335$.

45) Sas a d u. Kit a m ro, Nippon Naikagakkai Zasshi, 1926-27, 14, 736. 


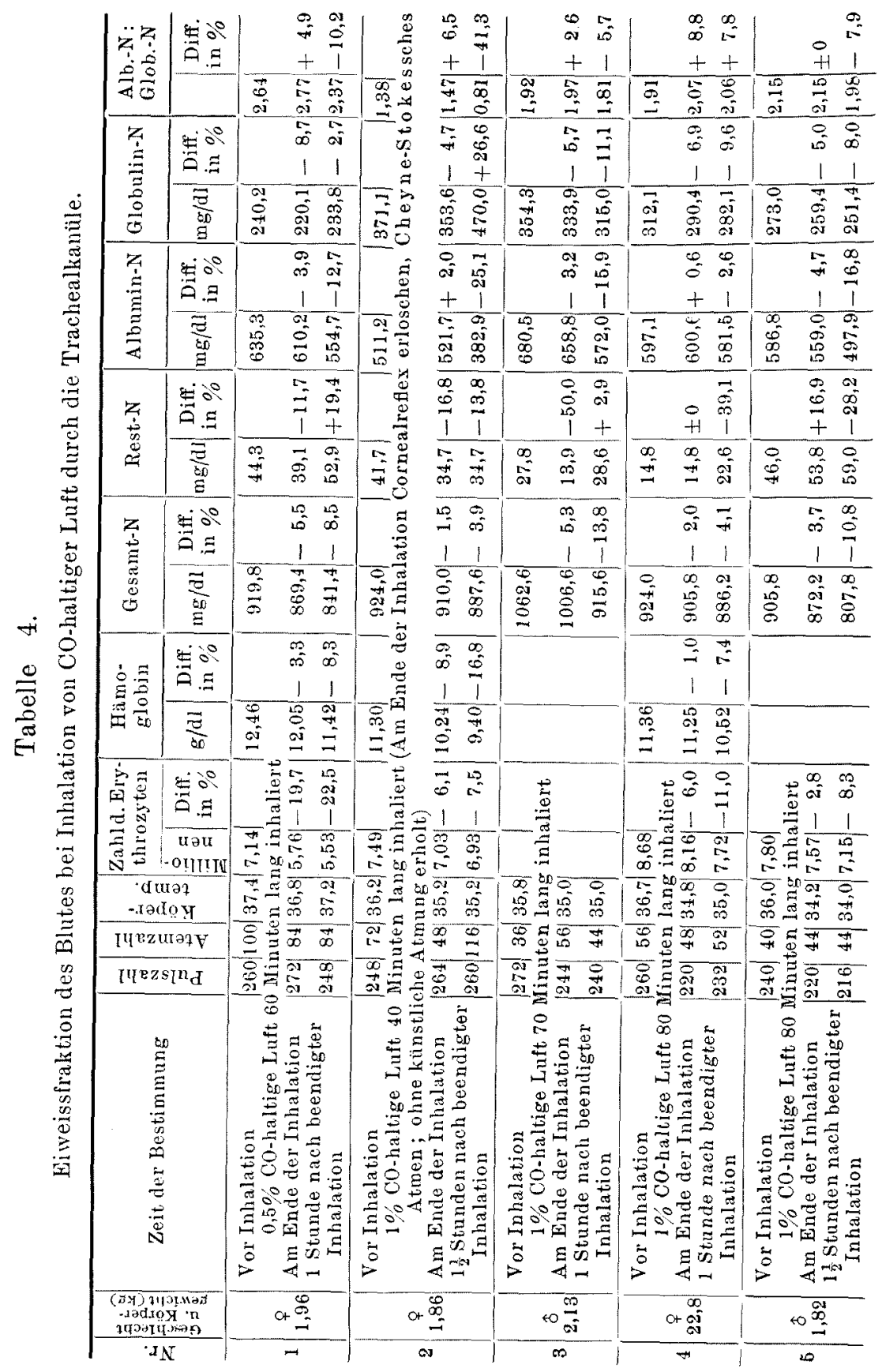




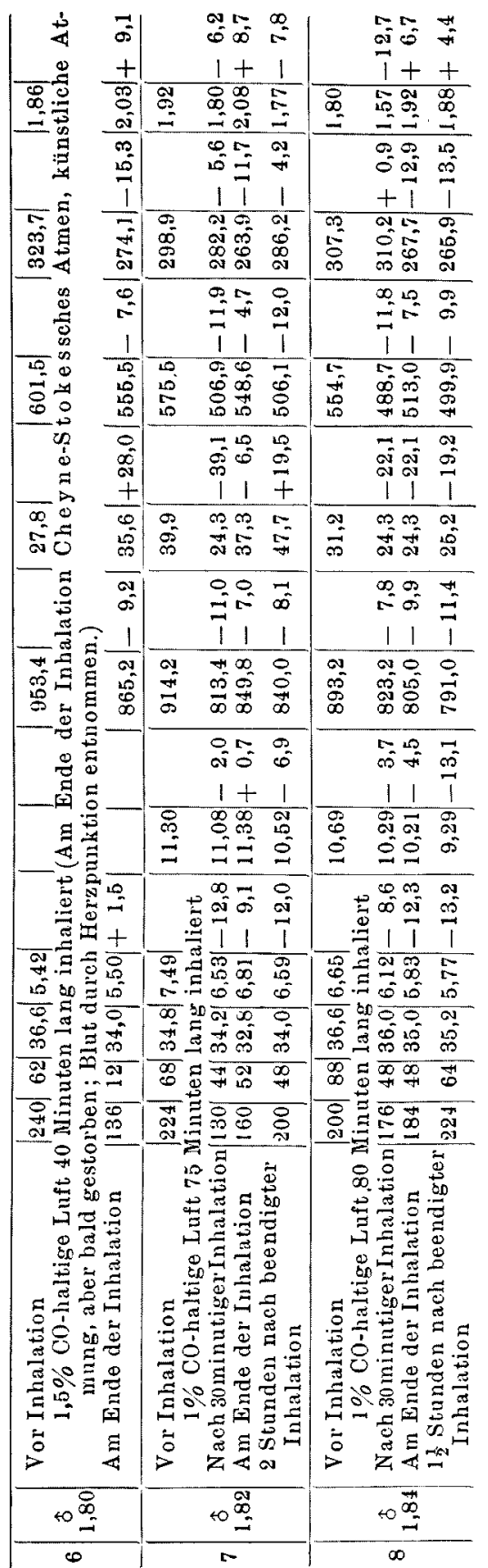

Wirkung Erythrozytenzahl, HbGehalt, Gesamt-N und Rest-N keine merkliche Veränderungen aufweisen. Auch in meiner Untersuchung verhielten sich eben besprochene Blutkomponenten nicht so deutlich abweichend von denselben im Kontrollversuch, aber in Anbetracht der Feststellung, dass in Versuch 6 wo, ad Exitus die Vergiftungssymptome sich dokumentierten, die Erythrozytenzahl, in wennauch geringem Masse, zunahm und ferner in Versuch 7, wo die Erythrozytenzahl, welche zuvor abgenommen hatte, hinterher zunahm, gewinnt man jedenfalls den Eindruck, dass bei fortschreitendem Vergiftungsprozesse etwa die Anhydrämie sich ereignen mag.

Der refraktometrisch bestimmte Serumeiweissgehalt, der im Anfangsstadium abgenommen hatte, vermehrte sich im fortgeschrittenen Stadium der CO-Vergiftung, worauf schon oben hingewiesen wurde. Beim Gesamt$\mathrm{N}$ indessen, wenn auch in Versuch 7 ähnliche Verhältnisse wie eben geschilderte sich kundgaben, war die Tendenz zur Zunahme im Endstadium der Vergiftung kaum wahrzunehmen. Dies duirfte wahrscheinlich davon hergerührt haben, dass durch die Anämie, welche durch Entziehungen grosser Blutmengen in kurzer Frist herbeigeführt wurde, Veränderungen, die sonst aufge- 
treten sein müssten, verdeckt wurden. Kasuga $\mathrm{i}^{24}$ hat durch Einatmenlassen von $\mathrm{O}_{2}$-armer Luft eine $\mathrm{Abnahme}$ des $\mathrm{Hb}$ und eine $\mathrm{Zu}$ nahme des Gesamt- $\mathrm{N}$ nachgewiesen.

Was nun das Verhalten des Rest-N anbelangt, nahm er, mit Ausnahme der 2 Fälle, durch die CO-Einatmung deutlich ab; eine Stunde nach Abschluss der Inhalation stieg der Rest-N selbstverständlich über den Wert hinaus, welcher im Laufe der Inhalation gefunden wurde, je sogar deutlich höher als der Präinhalationswert. Im übrigen ist zu bemerken, dass der Rest-N unter fortgesetzter Inhalation im Stadium, wo die Vergiftungssymptome noch nicht in den Vordergrund traten, niedrigere Werte zeigte als auf dem Gipfelpunkt der Vergiftung (Versuch 7-8).

Bütne $r^{6)}$ hat beim Versuch der Leuchtgasvergiftung an Kaninchen eine deutliche Abnahme des Rest- $\mathrm{N}$ gefunden; diese Abnahme will er davon abhängig gemacht wissen, dass durch den $\mathrm{O}_{2}$-Mangel die Muttersubstanz, aus welcher der Rest-N angeboten wird, nicht völlig oxydiert werden kömne. Der genannte Autor hat des weiteren die Beobachtung gemacht, dass dieser Abfall des Rest-N nur sehr kurz dauern kann, worauf derselbe dann auffallend ansteigen kann; dabei sprach er die Vermutung aus, dass derartige Zunahme entweder auf der Ausscheidungsstörung des Rest-N, die durch fortschreitende Nierenschädigung hervorgerufen wird, oder auf der überstürzten Bildung des Rest-N beruhe. In meiner Untersuchung wurde auch durch die Inhalation von CO-haltiger Luft unter Umständen die Zunahme des Rest-N, wie es in Versuch 5 und 6 der Fall war, beobachtet, was also bis zu einem gewissen Grad durch unterschiedliche Vergiftungsgrade bedingt sein dürfte. Immerhin dürfte der Rest-N durch die CO-Vergiftung zuerst abnehmen, dann aber, wenn die Vergiftung einen bestimmten Grad erreicht hat, im Verein mit der von Yagata ${ }^{4(5)}$ erwiesenen Funktionsschädigung der Niere eher zunehmen. Darauf diufte auch wohl beruhen die Feststellung, dass in Ver'such 7 und 8 der Abnahmegrad des Rest-N nach 60 Minuten lang anhaltender Inhalation kleiner oder gleich war wie der Abnahmegrad nach halbstiundiger Inhalation; und die Zunahme des Rest-N, welche nach Abschluss der Inhalation erfolgte, lässt sich leicht dahin erklären, dass der Organismus sich vom bis dahin bestandenen $\mathrm{O}_{2}$-Mangel erholt hat, wobei die Oxydationsprozesse naturgemäss lebhafter vonstatten gehen.

Nun möchte ich auf die Veränderungen der Eiweissfraktionen

46) Yagata, Jikken Shokakibyogaku, 1930, 5, 1100. 
eingehen: Alb.-N nahm in Versuch 1-6, wo die Bestimmungen nur am Ende der Inhalation vorgenommen wurden, um ein Geringes ab, in einem Fall (Versuch 2) aber umgekehrt zu. Globulin-N nahm aber stets ab, der Abnahmegrad war obendrein grösser als der des Alb.-N. Der Eiw.-Quot. war mithin erhöht. In Versuch 7-8, wo die Bestimmungen 30 Minuten nach beginnender Inhalation ausgeführt wurden, war Alb.-N in starker Abnahme begriffen, während Glob.-N zur selben Zeit nicht so erheblich abnahm, mitunter umgekehrt zunahm. Am Ende der Inhalation schlugen die Verhältnisse ins Gegenteil um, indem Alb.-N, welcher bis vorhin in erheblichem Masse abgenommen hatte, nunmehr die Tendenz zum Wiederanstieg. zeigte, während Glob.-N hingegen beträchtlich abnahm. Hieraus geht also hervor, dass der Eiw.-Quot. mit der Progredienz der Vergiftung zuvörderst erheblich absinkt und dann ansteigt.

Nach Ablauf von 30-120 Minuten nach Aufhören der Inhalation war Alb.-N, welcher im späteren Stadium der Intoxikation zur Zunahme geneigt war, von neuem merklich vermindert, und zu gleicher Zeit besass Glob.-N, wenn er auch in einigen Fällen noch weiter abnahm, doch in der Mehrzahl der Fälle die Tendenz zur Zunahme, wobei der Eiw.-Quot. dementsprechend zum mindesten im Vergleich zum Wert, der am Ende der Inhalation ermittelt wurde, stets erniedrigt war. Es ist dies als ein Ausdruck dafür aufzufassen, dass der Einfluss der Vergiftung erst fortzufallen im Begriffe ist und an deren Stelle lediglich die durch Blutentnahme bedingte Veränderung nunmehr sich geltend macht.

\section{Besprechung.}

Über die biologische Bedeutung der Bluteiweisskörper herrschen noch derzeitig manche Unklarheiten. Nach bisher errungenen Kenntnissen fällt den Bluteiweisskörpern die Aufgabe zu, sich am Lebensvorgang, als Nähr- und Baustoff des Körper's zu beteiligen; eine andere Aufgabe besteht darin, vermöge ihres kolloid-osmotischen Drucks für den durch die Kapillarwand hindurch zwischen Blut und Geweben sich abspielenden Stoffaustausch eine wichtige Rolle zu spielen.

Legt man sich die Frage vor, aus welcher Fraktion vom Bluteiweiss der in Betracht kommende kolloid-osmotische Druck hauptsächtlich stammt, so wissen wir aus Untersuchungen von Farkas ${ }^{47)}$

47) F a rk a s, Ztschr. f. exp. Med., 1926, 53, 666. 
und Beck man n, ${ }^{48)}$ dass das Fibrinogen keinen messbaren k. o. D. liefert. Wells ${ }^{49}$ u. a. vertreten die Ansicht, dass k. o. D., welchen der 1\%ige Eiweissgehalt ausübt, vorwiegend durch den Albumingehalt bestimmt werde, indessen durch Untersuchungen von Ruszny ak, ${ }^{50 j}$ Farkas, $\left.{ }^{47}\right)$ Beckmann $^{48}$ u. a. sowie von Oelkers ${ }^{51}$ ist dargelegt worden, dass die Veränderung des Dr. pro\% durch das AlbuminGlobulinmischverhältnis, also den Eiw.-Quot. beeinflusst werden können, und auch $\mathrm{Kasugai^{24 }}$ an hiesiger Klinik hat den Nachweis erbracht, dass die Schwankung des k.o. D.-Wertes der Verschiebung des Eiw.-Quot. immer parallel geht. Ich habe auch im Verlaufe der CO-Vergiftung bestätigt, dass der Umstand, dass k. o. D., der sich im Anfangsstadium der CO-Vergiftung zunächst herabgesetzt hat, mit der Progredienz der Vergiftung in die Höhe getrieben wird, mit dem Verhalten des Eiw.-Quot. zuerst in erheblichem Masse abzusinken und sich darauf auf das Anfangsniveau wiederherzustellen, in gutem Einklang steht. Es drängt sich dann die Frage auf, wovon denn derartige von mir erhobene Veränderung des Eiw.-Quot. herrühren würde.

Nach Versuchsergebnissen von den an hiesiger Klinik sich betätigenden Forschern, wie Saito, ${ }^{32}$ Horikawa, ${ }^{52}$ Tada u. Nakazawa, ${ }^{53)}$ Onozaki, ${ }^{54)}$ Yasuda, ${ }^{55}$ Fukuhara, ${ }^{56)}$ Kasugai, ${ }^{54}$ Sana$\mathrm{d} a{ }^{57)}$ unterliegen die Bildung und die Regulierung der Bluteiweisskörper unverkennbar den Einflüssen von der Leber, Niere, Milz, dem Retikuloendothelialsystem, Knochenmark und Zentralnervensystem, und es ist eine allbekannte Tatsache, dass bei akuter und chronischer CO-Vergiftung an oben genannten Organen sich rerschiedenste pathologisch-anatomische Veränderungen einstellen können.

$\mathrm{K}$ e n n w e ${ }^{58}$ machte die Beobachtung, dass bei der CO-Intoxikation neben der Hyperämie der Milz trübe Schwellungen der Glomeruli und sowie der Harnkanälchen der Niere auftreten. Cam pbel1 ${ }^{12}$ fand, dass durch die chronische CO.Vergiftung an der Leber und Herzmuskulatur venöse Staunng sowie Zellatrophie vorkommen. Yaga $\mathrm{ta}^{46}$ konnte durch die Untersuchung über das

48) Beckmann, Bass, Dürr u. Drasi n, Ztschr. f. exp. Med., 1926, 53, 420.

49) Wells, Y ou ma ns u. Miller, Journ. Clin. Invest., 1933, 12, 1103.

50) Ruszynyak, Ztschr. f. exp. Med., 1924, 41, 532.

51) Oelkers, Ztschr. f. kl. Med., 1931, 115, 854.

52) Horik a wa, Tohoku Journ. Exp. Med., 1936, 28, 90.

53) T ada u. N ak a za wa, Ibid., 1930, 15, 119.

54) Onozaki, rbid., 1935, 25, 131.

55) Y a suda, Nippon Naikagakkai Zasshi, 1935, 23, 343.

56) Fuk uha ra, Ibid, $1935,23,343$.

57) S a n ad a, Tohoku Journ. exp. Med., 1936, 28, 144.

58) Kennweg, Dtsch. Ztschr. ges. gerichtl. Med., 1922, 1, 423. 
Ausscheidungsvermögen der Farbstoffe bei der akuten CO-Vergiftung zeigen, dass das CO die Niere und die Leber angreift. Ramsey, Eilmann u. Toled $\mathrm{o}^{59}$ ) haben Beweis geliefert, dass es bei entsprechender Vergiftung an Leber, Milz und Niere zu Hyperämie und kleiner Blutung kommt. Abo ${ }^{60}$ u.a. sahen bei chronischer CO-Vergiftung das Retikuloendothelialsystem brühend wuchern, Nishimura ${ }^{61}$ ) hat an Milz, Leber und Niere die Staung sowie die Degeneration nachgewiesen.

Nach oben angeführten literarischen Angaben ist es einleuchtend, dass durch die CO-IVirkung gewisse Veränderungen nicht allein in der Eiweissbildung, sondern auch in der Mobilisation und Regulation des Eiweisses zustande kommen. Hinsichtlich der Verschiebung im Albumin-Globulin-Verhältnisse sind Schindera, ${ }^{62}$ Howe u. Sanderson ${ }^{63)}$ und Arnd u. Hafner ${ }^{64)}$ der Ansicht, dass sie eine unspezifische, mit dem Fieber u. a. identische Reaktion sei; indessen von vielen Forschern ist dargetan worden, dass das Albumin-Globulin-Verhältnis unter verschiedenen pathologischen Verhältnissen erfassbare Veränderung aufweist. $\mathrm{Ozawa} \mathrm{z}^{65}$ hat im Versuch an Hunden, indem er durch Iujektion von $\frac{1}{5}$ Normal-HCl oder durch die Kältereizung die Azidosis erzeugte, sowohl die Globulinvermehrung wie auch die Albuminverminderung nachgewiesen. Die gesteigerte Permeabilität der Gefässwand bei der Azidosis ist durch Untersuchungen von $\mathrm{Land}$ is ${ }^{66)}$ klargestellt worden, und nach $\mathrm{Mikami}{ }^{67)} \mathrm{Kamei}^{\mathrm{i}^{63}}$ und $\mathrm{Thie} \mathrm{l}^{69}$ tritt eine ausgesprochene Azidosis bei CO-Vergiftungein was jüngst auch von $\mathrm{mir}^{25)}$ experimentell nachgewiesen wurde.

Nach dem oben Gesagten ist mit grösster Wahrscheinlichkeit zu erwarten, dass unter dem Einfluss des Kohlenoxyds der Stoffaustausch zwischen Kapillaren und Geweben, mithin das Albumin-Globulin-Mischverhältnis unfehlbar irgendwie Veränderungen erleiden. Des weiteren ist schon oben darauf eingegangen worden, dass die Ansichten von verschiedenen Autoren über das Verhalten des Eiw.Quot. beim bei der CO-Vergiftung die wichtigste Rolle spielenden

59) Ramsey, Eilman n u. Toled o, Journ. Labor. a. Clin. Med., 1931-32, 17, 415.

60) Abo, Kinoshita, Nakamura u. O no, Nippon Byorigakkai Zasshi, 1932, $22,183$.

61) Nishimura, Kokumin Eisei, 1926-27, 4, 438.

62) Schindera, Dtsch. Arch. f. kl. Med., 1924, 114, 113.

63) Howe u. Sanderson, Journ. Biol. Chem., 1924-25, 62, 767.

64) Arnd u. Hafner, Biochem. Ztschr., 1926, 167, 440.

65) Ozawa, Fukuoka Ikadaigaku Zasshi, 1926, 19, 591 u. 701.

66) Landis, Amer. Journ. Physiol., 1928, 83, 528.

67) Mik a mi, Tohoku Journ. Exp. Med., 1927, 8, 236.

68) Ka mei, Ibid., 1931, 17, 107 u. 127.

69) Thiel, Ztschr. f. exp. Med., 1933, 88, 207. 
$\mathrm{O}_{\mathrm{s}}$-Mangel sehr divergieren, indem manche Autoren die Albuminvermehrung und andere die Globulinvermehrung im Blut annehmen.

Auf Grund der oben geschilderten literarischen Angaben und basierend auf meinen Versuchsergebnissen hat man wohl Grund anzunehmen, dass die bei akuter CO-Vergiftung auftretenden Veränderungen der Eiweissfraktionen nicht lediglich durch eine einzige Ursache ausgelöst werden, sondern dass es sich hierbei darum handelt, dass mancherlei Faktoren, wie die allgemeine Anoxämie, die dadurch bedingte Azidosis und die primäre toxische CO-Wirkung, indem sie alle im Kausalnexus gleichsam altruistisch mitwirken, Funktionsstörungen der eiweissbildenden und eiweissregulierenden Organe hervorrufen, wodurch nebst der Eiweisskonzentration des Blutes auch das Albumin-Globulin-Verhältnis in Mitleidenschaft gezogen wird. Das alles muss oben erwähnte Daten gezeitigt haben. In meiner anderen Mitteilung wird die Rede davon sein, dass für die Albuminvermehrung im moribunder Zustand des Versuchstieres die Leber die wichtigste Rolle spielt.

\section{Schluss.}

Bei 40-90 Minuten dauernder Einatmung von 0,5-1\% CO-haltiger Luft treten im Serum arteriellen Blutes des Kaninchens folgende physikochemische und chemische Veränderungen auf:

1. Serumeiweiss und dessen k. o.D. sowie Dr. pro\% nehmen zuerst in erheblichem Masse ab, in fortschreitendem, dem Tode nahen Stadium der Vergiftung vermehren sich alle zuvor verminderte Werte immer mehr und können mitunter die ursprünglichen Werte überschreiten.

2. Entsprechend den Veränderungen des kolloid-osmotischen Drucks, erniedrigt sich der Albumin-Globulin-Quotient, um dann aber anzusteigen. Zunächst nimmt das Albumin auffallend ab, schlägt aber mit der Progredienz der Vergiftungserscheinung in die Zunahme um, während das Globulin weiter stufenweise immer mehr abnimmt.

3. Rest-N vermindert sich zuerst, zeigt aber alsdann die Tendenz zu allmählicher Zunahme.

Herrn Dr. F. Nakazawa, a.o. Professor an hiesiger Klinik mächte ich hier meinen herzlichen Dank für seine freundliche Leitung bei dieser Arbeit aussprechen. 